\title{
Common Neural Substrates for Response Selection across Modalities and Mapping Paradigms
}

\author{
Yuhong Jiang ${ }^{1}$ and Nancy Kanwisher ${ }^{1,2}$
}

\begin{abstract}
In many situations, people can only compute one stimulusto-response mapping at a time, suggesting that response selection constitutes a "central processing bottleneck" in human information processing. Using fMRI, we tested whether common or distinct brain regions were involved in response selection across visual and auditory inputs, and across spatial and nonspatial mapping rules. We isolated brain regions involved in response selection by comparing two conditions that were identical in perceptual input and motor output, but differed in the complexity of the mapping rule. In the visualmanual task of Experiment 1, four vertical lines were positioned from left to right, and subjects pressed one of four keys to report which line was unique in length. In the auditory-manual task of Experiment 2, four tones were presented in succession, and
\end{abstract}

\section{INTRODUCTION}

Major research efforts in cognitive neuroscience have addressed the neural mechanisms underlying both the perceptual analysis of stimuli and the preparation of motor responses. However, successful interaction with the world requires that perception and action be linked. The present investigation focuses on the process of mapping stimuli onto responses (called "response selection" [RS]).

A substantial literature in cognitive psychology has suggested that RS represents a fundamental bottleneck in human information processing (for a review, see Pashler, 1994, 1998). Dual-task interference studies on choice RT tasks indicate that several perceptual processes can be conducted in parallel without interference (Treisman \& Davies, 1973), and several motor responses can be produced simultaneously without interference (Pashler, 1990), but under most conditions only a single stimulus-to-response ( $\mathrm{S}-\mathrm{R})$ mapping can be computed at a time (Pashler, 1984). Importantly, this "central processing bottleneck" is general across different modalities of input and output. Thus, while we can see a shape and hear a tone at the same time, and we can

\footnotetext{
${ }^{1}$ Massachusetts Institute of Technology, ${ }^{2}$ Athinoula A. Martinos Center for Biomedical Imaging, Charlestown
}

subjects pressed one of four keys to report which tone was unique in duration. For both visual and auditory tasks, the mapping between target position and key position was either spatially compatible or incompatible. In the verbal task of Experiment 3, subjects used nonspatial mappings that were either compatible ("same"' if colors matched; "different" if they mismatched) or incompatible (the opposite). Extensive activation overlap was observed across all three experiments for incompatible versus compatible mapping in bilateral parietal and frontal regions. Our results indicate that common neural substrates are involved in response selection across input modalities and across spatial and nonspatial domains of stimulus-to-response mapping, consistent with behavioral evidence that response selection is a central process.

press a key and say a word at the same time, we cannot simultaneously determine which key to press on the basis of the shape and which word to say on the basis of the tone. Exceptions to this rule occur under some circumstances: when the S-R mapping is highly compatible (Pashler, Carrier, \& Hoffman, 1993), after substantial practice (Schumacher et al., 1999; Schumacher, Seymour, Glass, Kieras, \& Meyer, 2001), or when subjects are encouraged to adopt a "daring" response strategy (Schumacher et al., 2001). While the implications of these exceptions on the nature of the bottleneck is subject to debate (Ruthruff, Pashler, \& Klaassen, 2001; Schumacher et al., 1999, 2001; Meyer et al., 1995; Meyer \& Kieras, 1997; Pashler, 1994), the persistence of dual-task interference is a robust finding.

Despite considerable behavioral evidence that RS may form a central processing bottleneck, this property of human cognition has been largely ignored by researchers in cognitive neuroscience. Indeed, when considered from a neuroanatomical perspective, the very idea of the central bottleneck represents a puzzle. It seems reasonable to assume that two processes can interfere with each other only if they engage common neural mechanisms (or different but interacting mechanisms). But what neural mechanisms are shared (or interact) between the two tasks of pressing a key based on a visual shape, and uttering a word based on a tone? 


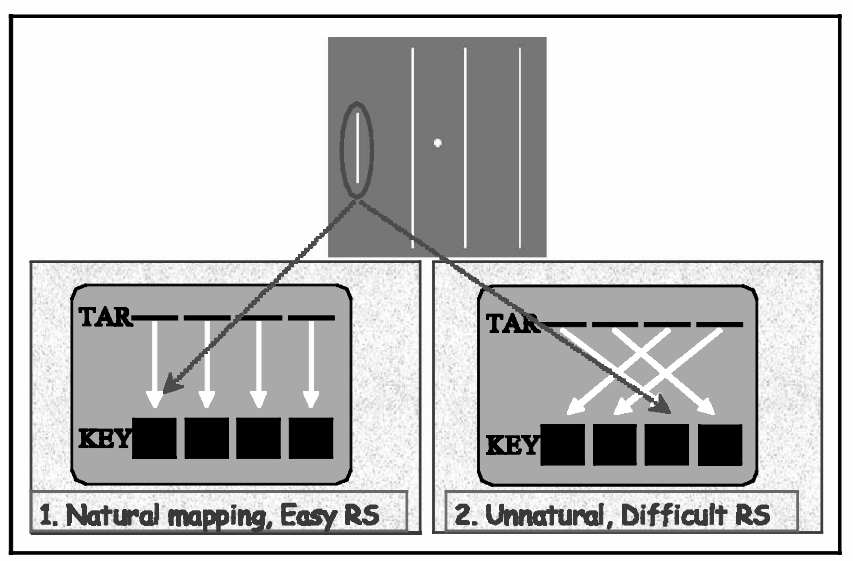

Figure 1. Sample display and the $S-R$ mapping rules for the visual-manual task. The task was to pick out the line with unique length and report its spatial position among the four lines. Target location was mapped to response location according to either natural mapping (left) or unnatural mapping (right). The same mapping rules (natural and unnatural mapping) were used in the auditory-manual task, in which subjects reported the temporal position of a unique tone among four tones.

If RS is a central cognitive process, then RS involving different input and output modalities should share common neural substrates. This hypothesis is tested here by asking whether there are brain regions that are activated in common by different kinds of RS, independent of input and output modalities. A companion article (Jiang \& Kanwisher, 2003) addresses a second prediction based on the central bottleneck view (Pashler, 1989), that some regions involved in RS should not also be involved in perceptual processing.

This study tests whether common regions are involved in various kinds of RS by asking three questions. First, what brain regions are engaged in RS in a visualmanual task? Second, are these regions specific to the visual modality, or are they also involved in RS for auditory stimuli? Third, are any common brain regions that are recruited in both visual and auditory RS engaged only when the two tasks share manual output or spatial mapping requirements? Although a few prior investigations of response interference in Stroop and other tasks have addressed the first question (Barch et al., 2001; Dassonville et al., 2001; Connolly, Goodale,
Desouza, Menon, \& Vilis, 2000; Hazeltine, Poldrack, \& Gabrieli, 2000; Botvinick, Nystrom, Fissell, Carter, \& Cohen, 1999; Carter et al., 1998; Carter, Botvinick, \& Cohen, 1999; Iacoboni, Woods, \& Mazziotta, 1996; Pardo, Pardo, Janer, \& Raichle, 1990), few if any prior studies have investigated the second and third questions (Schumacher \& D'Esposito, 2002). Based on the behavioral literature, we predicted that RS based on different input modalities, mapping paradigms (whether spatial or not), and output modalities should recruit common brain regions.

\section{RESULTS}

\section{Behavioral Performance}

Figure 1 illustrates the $\mathrm{S}-\mathrm{R}$ mapping rules used in Experiments 1 and 2 . Responses were either spatially compatible or incompatible with the stimuli. Table 1 shows mean RT and accuracy collected during scanning in the three experiments. In all experiments, incompatible mapping was substantially slower than compatible mapping. In the auditory-manual task, it was also less accurate. Thus, we were successful in manipulating the difficulty of $\mathrm{S}-\mathrm{R}$ mappings (i.e., RS) in all experiments.

\section{fMRI Data from the Visual-Manual Task}

We split data from the visual RS task into two independent halves, and carried out a random effects analysis on one half to generate group regions of interest (ROI) involved in visual-manual RS. The percent signal change (PSC) within these ROIs was then measured in the other half of the visual RS data, in the auditory-manual RS data, and in the visual-verbal RS data.

\section{Random Effects Analysis}

A random effects analysis was conducted on one half of each subject's data $(N=17)$, searching for any brain regions that had higher BOLD for the spatially incompatible mapping than compatible mapping $(p<.001$, uncorrected). This analysis revealed (see Figure 2) bilateral parietal activation that followed the intraparietal sulcus (IPS) and extended to the superior parietal lobule

Table 1. Behavioral Performance during Scanning

\begin{tabular}{|c|c|c|c|c|c|c|c|c|}
\hline \multirow[b]{2}{*}{ Experiment } & \multicolumn{4}{|c|}{ Accuracy (\%) } & \multicolumn{4}{|c|}{$R T$ (msec) } \\
\hline & Unnatural & Natural & $S E$ & $p$ & Unnatural & Natural & $S E$ & $p$ \\
\hline 2. Auditory RS & 76 & 82 & 2 & .01 & 717 & 544 & 22 & .0001 \\
\hline 3. Verbal naming & & N/A & & & 511 & 371 & 24 & .0001 \\
\hline
\end{tabular}

$\mathrm{RS}=$ response selection; $S E=$ standard error; $n s=$ nonsignificant; N/A $=$ not applicable. 
Figure 2. Regions of the brain that showed higher BOLD during unnatural than natural mapping between a visual target and a manual response $(n=17, p<.001$, random effects uncorrected; all the significant voxels are shown in red). The green arrows point to voxels containing most significant voxel of the ROI. These ROIs include: 1. Left anterior IPS (LaIPS); 2. Left posterior IPS (LpIPS); 3. Right anterior IPS (RaIPS); 4. Right posterior IPS (RpIPS); 5. Right precuneus; 6. Left frontal eye field (LFEF);

7. Right frontal eye field (RFEF); 8. Left inferior frontal gyrus (LGFi); 9. Left middle frontal gyrus (LGFm); 10. Right middle frontal gyrus (RGFm); 11. Left operculum; 12. Right operculum; 13. Right cerebellum. Left hemisphere is shown on the left side of the figure.

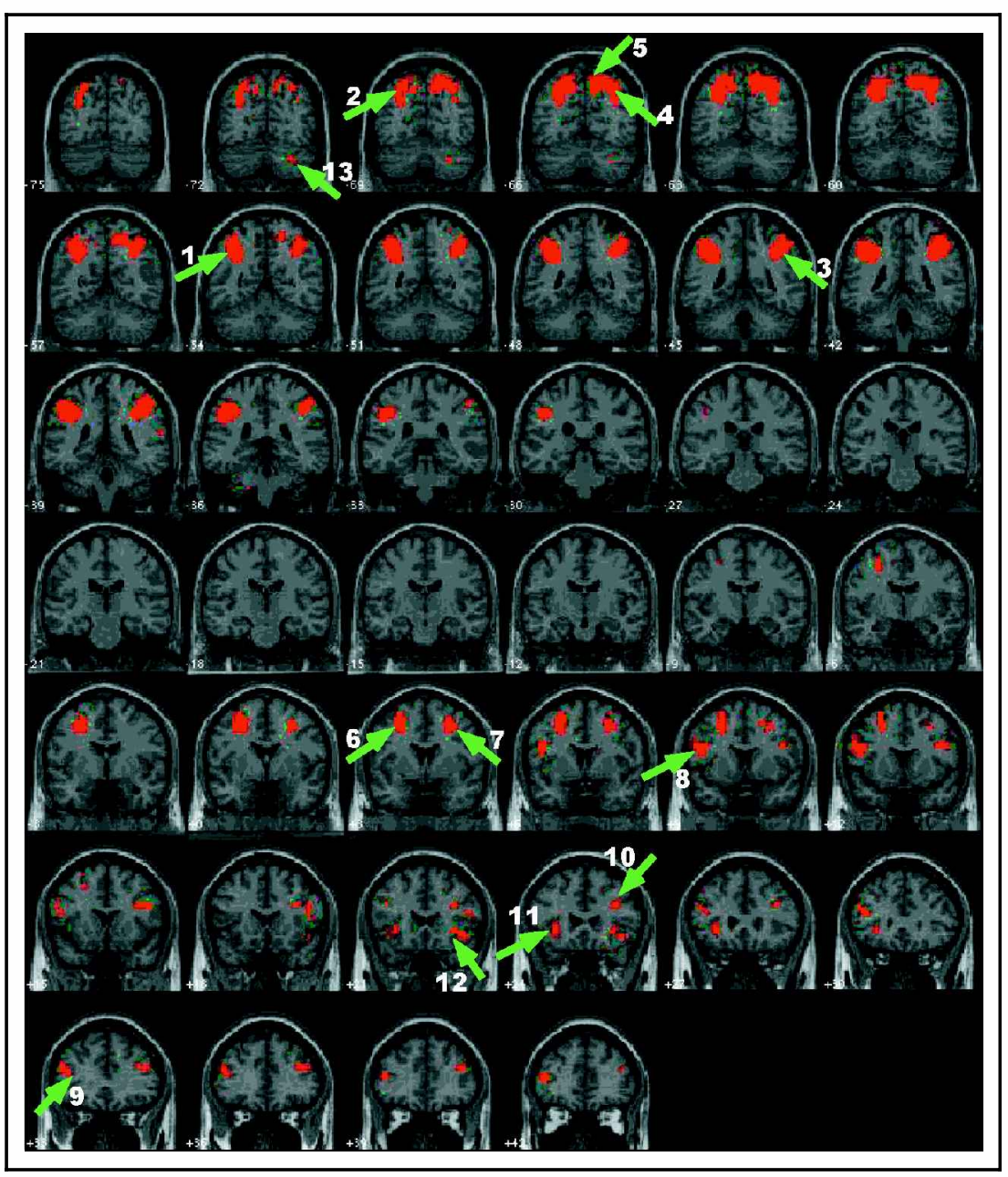

bilaterally and to the precuneus in the right hemisphere, bilateral frontal eye fields (FEFs), bilateral inferior/middle frontal gyrus surrounding area $46(\mathrm{GFi} / \mathrm{GFm})$, bilateral opercular/insula regions surrounding area 44/47 (frontal operculum/insula), and right cerebellum. Because activation in the IPS covered a large anatomical region which may not be functionally homogeneous, we subdivided it into an anterior and a posterior volume in the ROI analysis discussed below. Group ROIs were defined functionally as regions that were activated by the incompatible $>$ compatible mapping contrast in the random effects analysis $(p<.001$, uncorrected for multiple comparison; see Table 2).

\section{Validation of the Visual-Manual RS Activation in an ROI Analysis}

To confirm that the activations we saw in the random effects analysis were replicable, we calculated within the group ROIs the PSC relative to fixation in the other half of the data. These PSC results are statistically independent from the data used to define the ROIs. Table 3 shows the PSC for each of the ROIs.
All parietal ROIs, the FEF, the left GFm, GFi, operculum, and the right cerebellum showed a significantly higher PSC in the incompatible than in the compatible mapping conditions in this analysis of the second half of the data, thus independently validating the significance of this effect in each of these ROIs in a fashion that avoids the statistical problems with multiple voxelwise comparisons. Activity in the right GFm and right operculum failed to reach significance on this stringent test, suggesting that their involvement in the RS task was less robust. However, the failure to reach significance in these ROIs in the second analysis is subject to type II error. In sum, the split-half ROI analysis showed that the majority of the ROIs showed significant activation for RS, and that a fronto-FEF-parietal network is engaged during the visual-manual mapping task.

\section{fMRI Data on the Auditory-Manual Task}

One primary piece of evidence that the processing bottleneck resides at a central cognitive level comes from dual-task studies involving inputs from different modalities (Pashler, 1990). Although visual and auditory 
perception can proceed in parallel (Duncan, Martens, \& Ward, 1996; Treisman \& Davies, 1973), strong interference occurs when two responses are selected, one based on visual and the other based on auditory input (Borger, 1963; Pashler, 1994). Thus, RS for stimuli presented in different input modalities relies on the same cognitive mechanism. A natural prediction from these behavioral findings is that common brain regions should be activated whether RS is based on visual or auditory input. Fourteen subjects who were tested in the visual - manual mapping task were also tested in an auditory-manual mapping task. The same mapping rules_compatible and incompatible-were used in both visual and auditory tasks.

\section{Random Effects Analysis on the Auditory-Manual Mapping Experiment}

We analyzed each subject's data individually and then performed a random effects analysis across subjects ( $p<.005$, uncorrected; $N=14$ ). The SPM map of auditory RS was qualitatively similar to the map of visual RS. Figure 3 shows the activation for visual and auditory modalities within the same 14 subjects, revealing that the two tasks produced largely overlapping activation (shown in green).

The overlap in the parietal and FEF ROIs was striking. Visual and auditory RS appear to rely on the same regions in these ROIs. In addition to the similarities, there were also apparent differences. For example, the inferior/ middle frontal gyri were significant for the visual but not the auditory RS task, while the thalamus/basal ganglia and the cingulate cortex showed the reversed pattern. Such differences may be attributed partly to threshold setting, such that one contrast passed the threshold while the other just missed it. Consistent with this hypothesis, an interaction test between modality and mapping rule failed to reveal any region that was activated more by one modality than the other. In addition, ROI analysis on regions that did not show overlap suggests that the trend of an incompatibility effect was the same for the visual and the auditory RS. Thus, the brain regions involved in RS appeared to be largely independent of input modality, at least for visual and auditory stimuli.

\section{ROI Analysis: Are Visual RS ROIs Also Involved in Auditory RS?}

To characterize the functional properties of the ROIs defined by visual RS in Experiment 1, we measured the PSC within these group ROIs in the auditory RS task, and in the second half of the visual RS task. Thus, both data sets were independent of the visual RS data used to define these ROIs. Table 4 shows the mean PSCs in the auditory - manual and visual - manual mapping tasks.

The regions that were strongly involved in visual RS the parietal ROIs and the FEFs - were also strongly involved in auditory RS. The regions whose activation in visual RS was weaker or failed to reach significancethe GFm and the operculum - also showed a weaker activation pattern in auditory RS. Thus, brain activations for RS were qualitatively similar across input modalities.

An ANOVA on modality (visual vs. auditory) and mapping rule (compatible vs. incompatible) within each

Table 2. Regions Showing Significant BOLD Activation for Unnatural > Natural Mapping in the Visual-Manual Mapping Task $(n=17$, random effect, $p<.001$, uncorrected $)$

\begin{tabular}{|c|c|c|c|c|c|}
\hline 900 & $-36-5443$ & $\mathrm{~L}$ & anterior IPS (area 40) & 9.59 & 6 \\
\hline \multirow[t]{2}{*}{775} & $42-4545$ & $\mathrm{R}$ & anterior IPS (area 40) & 7.33 & 12 \\
\hline & $30-6648$ & $\mathrm{R}$ & posterior IPS (area 7) & 6.78 & 12 \\
\hline 222 & -27348 & $\mathrm{~L}$ & FEF (area 6) & 5.36 & 12 \\
\hline 92 & 33348 & $\mathrm{R}$ & FEF (area 6) & 5.39 & 12 \\
\hline 182 & -48921 & $\mathrm{~L}$ & GFi (area 44/46) & 5.02 & 12 \\
\hline 40 & $4821-12$ & $\mathrm{R}$ & operculum (area 44/47) & 5.22 & 12 \\
\hline 19 & $33-72-33$ & $\mathrm{R}$ & cerebellum & 4.28 & 12 \\
\hline
\end{tabular}

MNI = Montreal Neurological Institute; $\mathrm{L}=$ left; $\mathrm{R}=$ right. 
Table 3. Percent Signal Change above Fixation for Each ROI in the Natural and Unnatural Mapping Conditions in the Second Data Set of the Visual-Manual Task, and $p$ Level of the $t$ Test for Unnatural Versus Natural Mapping across Subjects

\begin{tabular}{|c|c|c|c|c|c|c|}
\hline & \multicolumn{3}{|c|}{ Left Hemisphere ROI } & \multicolumn{3}{|c|}{ Right Hemisphere ROI } \\
\hline & Natural & Unnatural & $p$ & Natural & Unnatural & $p$ \\
\hline aIPS & 0.17 & 0.29 & $* *$ & 0.08 & 0.25 & $* * *$ \\
\hline pIPS & 0.10 & 0.27 & $* * *$ & 0.10 & 0.26 & $* * *$ \\
\hline FEF & 0.22 & 0.37 & $* * *$ & 0.16 & 0.27 & $* * *$ \\
\hline GFm & -0.02 & 0.04 & + & -0.05 & 0.00 & $n s$ \\
\hline Operculum & 0.00 & 0.06 & + & -0.03 & 0.07 & $n s$ \\
\hline Precuneus & & $\mathrm{N} / \mathrm{A}$ & & 0.07 & 0.29 & $* * *$ \\
\hline GFi & 0.09 & 0.22 & $* * *$ & N/A & & \\
\hline Cerebellum & & N/A & & 0.19 & 0.27 & + \\
\hline
\end{tabular}

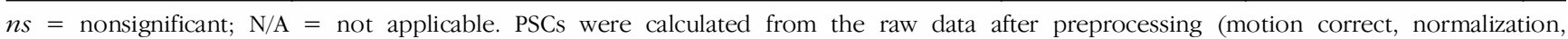
and smoothing).

${ }^{+} p<.10$.

$* * p<.01$.

$* * * p<.001$.

ROI showed that the PSCs were generally higher during the auditory task than the visual task for both compatible and incompatible mappings. This main effect of modality was significant in all ROIs except in the bilateral posterior IPS and right GFm. This modality effect may be due to the fact that it was harder to map an auditory sequence onto the keys than to map a visual array onto the keys, reflected by subjects' self-report of effort. The main effect of mapping rule was significant in all the ROIs except the bilateral GFm and the right operculum. For the majority of the ROIs, the interaction between modality and mapping rule was not significant, suggesting that visual and auditory RS produced similar activation. The only ROI that showed a significant interaction was the left anterior IPS, $p<.01$, where higher RSrelated activity was found for the auditory than the visual modality. Even in this ROI, however, both visual and auditory RS were significant, suggesting that the left aIPS was not restricted to processing RS from one modality only.

The similarity in activations between visual and auditory RS cannot be attributed to the selection of group ROIs that averaged across many different brains. In a further analysis, we selected ROIs based on each individual subject's visual RS, and replicated the results reported above. Thus, both the random effects SPM analysis and the ROI analysis showed that RS based on visual and on auditory inputs involved similar regions of the brain. Further, when ROIs were defined based on their activity in the auditory RS task, those which showed a significant effect for the auditory task were also significant for the visual task $(p s<.05)$. These findings support the hypothesis that RS constitutes a central process occurring after perceptual processing.
Comparing the left and right ROIs for the visual and the auditory RS task, we found a significant difference between modalities in the laterality of parietal activation, $p<.05$. Whereas the RS effect was larger in the left than in the right IPS for the auditory modality, it was larger in the right than in the left for the visual modality. However, note that the RS effect was significant on both left and right IPS in both modalities. Thus, although RS activities were not identical across visual and auditory modalities, they were largely modality independent.

\section{fMRI Data from the Verbal Naming Task}

In the verbal naming task, we tested whether the regions involved in a spatial visual-manual RS task were also involved in a nonspatial, verbal RS task. The purpose of this experiment was to test the generality of the regions for RS found earlier in the manual tasks. Specifically, we sought to test whether the activity we saw in the visual and auditory RS tasks might be due to the fact that both tasks required spatial mapping as well as manual responses. In this task, subjects decided whether two patches of color - presented successively at the center of the screen-were the same or different. In the compatible mapping condition, they responded "same" verbally if the colors matched and "different" if the colors did not. In the incompatible mapping condition, they made the opposite response. The response mapping here was thus completely nonspatial, and the motor output was verbal. If the common activation between visual and auditory RS was specific to spatial mapping between stimulus space and motor space or specific to making manual responses, they should not be involved in the verbal naming task. 
Figure 3. Overlapping activation (green) across visual (red) and auditory (blue) response selection in a random effects group analysis ( $n=14, p<.005$, uncorrected).

\section{Head Motion}

Although most fMRI studies have avoided the use of overt speech during scanning because of concerns about head motion, recent studies suggest that meaningful data can be obtained while subjects speak aloud (Barch, Braver, Sabb, \& Noll, 2000; Huang et al., 2002; Palmer et al., 2001; Rosen, Ojemann, Ollinger, \& Petersen, 2000; Barch et al., 1999). In our verbal naming task, subjects were instructed to make an overt verbal response while minimizing lip and mouth movements. For the two scans involving overt naming, the overall amount of motion calculated by SPM motion correction was below $0.5 \mathrm{~mm}$ in each of the three translations and below $0.01^{\circ}$ in each of the three rotations. The amount of motion during the two scans involving overt verbal response was not significantly larger than that during the two preceding scans without overt speech (e.g., during auditory RS).

\section{Random Effects Analysis on Verbal Naming}

A random effects analysis was performed on the group data for the verbal naming task $(N=12 ; p<.005$, uncorrected; unnatural $>$ natural naming). Figure 4 shows the voxels that reached significance, with the visual RS activation from a different group of 12 subjects overlaid.

The activation for the naming task in parietal regions was largely overlapping with the visual RS task, although it did not extend as far posteriorly. Overlapping activation was also observed in the FEF, the GFi/GFm, and the operculum/insula. In addition, resolving interference in verbal naming activated the paracingulate sulcus region (pre-SMA [9 21 48], Rushworth, Hadland, Paus, \& Sipila, 2001). Activation unique to the naming task can also be seen near the fusiform gyrus, which has been reported to be involved in color processing (e.g., Tootell \& Hadjikhani, 2001; Beauchamp, Haxby, Jennings, \& De Yoe, 1999). These differences were tested in a mapwise interaction between experiment and compatibility. The visual-manual task more significantly activated left anterior IPS [-39 -54 45], while the verbal-color task more significantly activated middle temporal gyrus $[-570-30 ;-4533-15]$, the fusiform gyrus $[-39-51-21 ; 39-51-15]$, and the anterior medial frontal regions [9 42 36; 9516 6]. An ROI analysis 
based on these regions showed that left anterior IPS was involved in both visual and verbal RS, while the other ROIs failed to reach significance for the incompatibility effect in either visual or verbal RS. Thus, it is unlikely that the regions that showed interaction in the mapwise comparison were involved in RS for only one modality.

\section{ROI Analysis: Are Visual-Manual ROIs Limited to Spatial S-R Mapping or Manual Responses?}

To test the functional properties of the group ROIs defined in the visual-manual task, here we measured the PSC in the natural and reversed verbal naming tasks within the group ROIs defined in Experiment 1. We also measured PSC within the group ROIs from a subset of 12 subjects from Experiment 1. The same number of subjects $(N=12)$ were selected in the two tasks so that they had comparable statistical power. The ROIs were the ones defined in Experiment 1. Table 5 shows the results.

Twelve of the 13 ROIs defined by their visual-manual RS activity were also significantly involved in the verbal naming task; the only exception was the right cerebellum, which may not be covered to the same extent across subjects. As in the random effects analysis, the ROI analysis revealed common activation between the visual RS and the verbal naming task. Some differences between the two tasks were also noted. For example, in the verbal naming task, the posterior IPS and the right precuneus showed signal lower than the fixation baseline. This may be related to the finding that the midline posterior brain, including the posterior cingulate and the precuneus, typically shows negative signals during demanding cognitive tasks (Gusnard \& Raichle, 2001; Raichle et al., 2001; Shulman et al., 1997). That the signal was above fixation during the visual-manual task suggests that in addition to their possible involvement in RS, these regions are also sensitive to spatial processing (Berman et al., 1999; Labar, Gitelman, Parrish, \& Mesulam, 1999; Culham et al., 1998). When the experiment (verbal naming vs. visual-manual mapping) was entered in an ANOVA as a between-subject factor, we failed to find a significant interaction between the experiment and mapping condition in any of the ROIs, suggesting that activity in these ROIs was not specific to spatial or nonspatial RS and was not specific to manual responses.

\section{Activation in the Anterior Cingulate Cortex across Experiments}

The anterior cingulate cortex (ACC) has been implicated in monitoring response conflict (Barch et al., 2001; Van Veen, Cohen, Botvinick, Stenger, \& Carter, 2001; Leung, Skudlarski, Gatenby, Peterson, \& Gore, 2000; Carter et al., 1998) and a priori it appears to be a likely candidate for the central processing bottleneck. However, in this study, the ACC failed to pass the threshold in

Table 4. Percent Signal Change above Fixation within the Visual RS ROIs in the Auditory-Manual and the Visual-Manual Mapping Tasks

\begin{tabular}{|c|c|c|c|c|c|c|c|c|}
\hline & \multicolumn{4}{|c|}{ Left Hemisphere ROI } & \multicolumn{4}{|c|}{ Right Hemisphere ROI } \\
\hline & \multicolumn{2}{|c|}{ Auditory-Manual } & \multicolumn{2}{|c|}{ Visual-Manual } & \multicolumn{2}{|c|}{ Auditory-Manual } & \multicolumn{2}{|c|}{ Visual-Manual } \\
\hline & Natural & Unnatural & Natural & Unnatural & Natural & Unnatural & Natural & Unnatural \\
\hline aIPS & 0.29 & $0.48 * * *$ & 0.12 & $0.18^{+}$ & 0.26 & $0.37 * *$ & 0.04 & $0.19 * * *$ \\
\hline pIPS & 0.09 & $0.30 * * *$ & 0.07 & $0.19 *$ & 0.08 & $0.21 * * *$ & 0.05 & $0.20 * * *$ \\
\hline FEF & 0.42 & $0.60 * * *$ & 0.24 & $0.38 * * *$ & 0.25 & $0.40 * *$ & 0.12 & $0.24 * * *$ \\
\hline GFm & 0.25 & $0.32 n s$ & -0.08 & $0.00 n s$ & 0.02 & $0.04 n s$ & -0.06 & $-0.07 n s$ \\
\hline Operculum & 0.34 & $0.39 n s$ & 0.01 & $0.06 n s$ & 0.26 & $0.32 n s$ & -0.09 & $-0.03 n s$ \\
\hline Precuneus & & & & & 0.25 & $0.49 * * *$ & 0.06 & $0.28 * * *$ \\
\hline GFi & 0.51 & $0.61 *$ & 0.07 & $0.19 * *$ & & & & \\
\hline Cerebellum & & & & & 0.49 & $0.61 * *$ & 0.24 & $0.34 * *$ \\
\hline
\end{tabular}

$n s=$ nonsignificant; $\mathrm{N} / \mathrm{A}=$ nonapplicable.

${ }^{+} p<.10$.

$* p<.05$.

$* * p<.01$

$* * * p<.001$. 
Figure 4. Overlapping activation (in green) between the visual RS task of Experiment 1 (in red) and the verbal naming task (in blue).

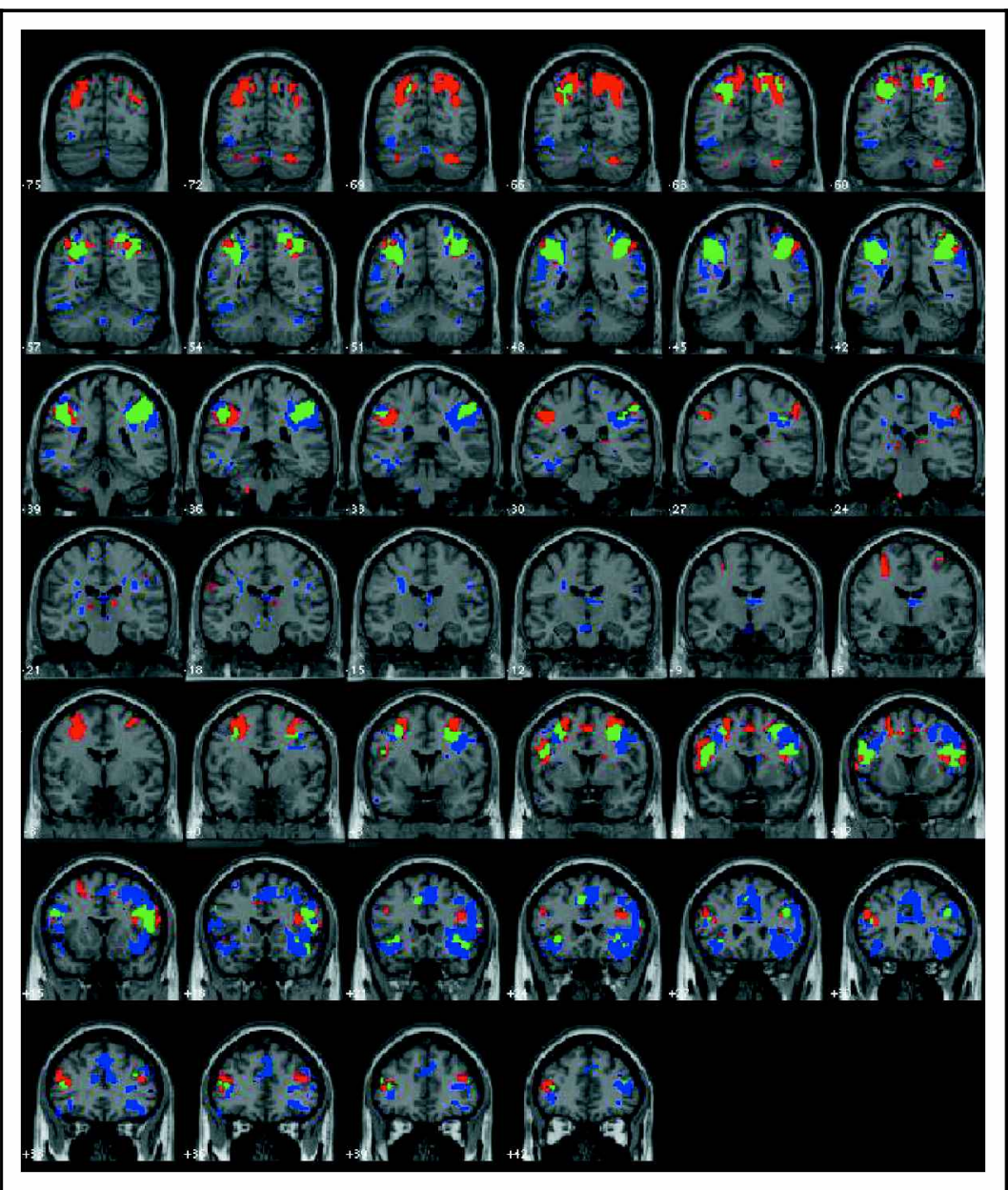

Experiment 1 and was not further tested. To examine activity in the ACC, here we defined an anatomical ROI centered on the ACC $([0,33,30]$, Van Veen et al., 2001). It included a spherical volume of 33 voxels with a radius of $6 \mathrm{~mm}$.

The ACC failed to be activated by the visual-manual mapping task $(-0.07 \%$ signal change for natural mapping, $-0.04 \%$ signal change for unnatural mapping, $p>.25)$, or by the verbal color matching task $(-0.10 \%$ signal change for natural report, $0.05 \%$ signal change for reversed report, $p>$.10). Interestingly, the ACC activation in the auditory RS task approached significance (0.07\% for natural mapping, $0.17 \%$ for unnatural mapping, $p<.053$ ). Past studies have argued that the ACC plays an important role in monitoring potential conflict, which correlates with error monitoring, but is not identical to error detection (Botvinick et al., 1999; Carter et al., 1998, 1999). In all three tasks, conflict monitoring is necessary, and may be greatest in the auditory RS task, reflected by a significant accuracy effect. This hypothesis about conflict monitoring explains activation in the auditory RS task, but does not account for the lack of activation in the other two experiments. Such lack of consistent ACC involvement in different kinds of RS argues against the ACC as the cognitive processing bottleneck.

\section{Negative Activation}

It is not uncommon to observe decreased BOLD signals associated with a more effortful task in certain brain areas (Raichle et al., 2001). Comparing the compatible and incompatible visual-manual mapping in a random effects analysis, a decreased BOLD signal for the incompatible mapping condition was seen in several regions typically associated with decreased activation: the cuneus/precuneus [9 -90 27], the posterior cingulate cortex [ 6 - 51 24], the superior frontal gyrus [24 39 51], the medial frontal cortex [-12 66 19], and the superior/ middle temporal gyrus [57-6-18]. In the auditorymanual mapping task, the same subtraction led to negative activation in the superior temporal gyrus [-39-12 9]. In the verbal naming task, negative activation was seen in the precuneus $\left[\begin{array}{lll}-6 & -51 & 33\end{array}\right]$, the posterior cingulate cortex $\left[\begin{array}{lll}-3 & -30 & 48\end{array}\right]$, the superior frontal gyrus [ $\left.\begin{array}{lll}-21 & 42 & 42\end{array}\right]$, the medial frontal cortex $\left[\begin{array}{lll}-6 & 57 & -3\end{array}\right]$, and the middle temporal gyrus [30 0 -33]. In all these "deactivated" regions, the PSCs for 
both natural and unnatural mapping were below fixation, with the unnatural mapping more negative. Decreased BOLD in these regions may be related to reduced self-referencing mental activity during demanding cognitive tasks (Raichle et al., 2001).

\section{DISCUSSION}

We used fMRI to identify brain regions engaged during RS, a process thought to represent a central bottleneck in human information processing (Pashler, 1984, 1994, 1998; see also Schumacher et al., 1999, 2001; Meyer et al., 1995; Meyer \& Kieras, 1997). The regions that produced significantly higher BOLD signal during incompatible versus compatible $\mathrm{S}-\mathrm{R}$ mappings across tasks include the bilateral IPS, extending into the SPL and the right precuneus, the bilateral FEFs, the right cerebellum, and less robustly, into the lateral frontal regions.

These regions support two properties of the hypothesized central processing bottleneck (Pashler, 1994): its independence from perceptual input and from motor effector. First, we found that largely overlapping regions of the brain were recruited when subjects selected responses based on visual and auditory inputs. Second, we observed common activation of a set of regions for RS across tasks involving manual output and overt speech. Finally, the similarity in activation between the visual-manual task and the verbal response task also indicates that this activation was not restricted to spatial mapping. These findings are con- sistent with the hypothesis that RS is a central cognitive process.

\section{Relationship to Prior Studies of RS}

Broadly defined, RS includes any process involved in mapping a stimulus onto a response. A number of possible subprocesses satisfy this criterion. In the visual-manual mapping task, for example, increasing the complexity of the mapping rule leads to increased demand on any of several related subprocesses: (1) $\mathrm{S}-\mathrm{R}$ mapping; (2) inhibition of prepotent responses based on the natural mapping rule; and (3) spatial remapping between the target space and the response space. Increasing the complexity of the mapping rule also increases the difficulty of the task. The selection of this task was guided by the behavioral literature, which has shown that a more complex mapping rule occupies the central bottleneck for a longer time (Pashler, 1994; McCann \& Johnston, 1992).

Another study (Jiang \& Kanwisher, in preparation) tested whether the activation we saw was related to S$\mathrm{R}$ mapping. That study tested 12 subjects in a further experiment that did not involve inhibition of prepotent responses or spatial remapping. Subjects were presented with geometric shapes - a square or a circle - and made either consistent responses to these shapes (e.g., one key for square and another for circle) or noncontingent responses (repeatedly keypresses independent of the shape). Thus, contingent minus noncontingent responses tested the presence of RS, uncontaminated by

Table 5. Percent Signal Change above Fixation in the Natural and Reversed Naming, and in the Natural and Unnatural Visual RS in the Group ROIs Defined by the Visual-Manual Task

\begin{tabular}{|c|c|c|c|c|c|c|c|c|}
\hline & \multicolumn{4}{|c|}{ Left Hemisphere ROI } & \multicolumn{4}{|c|}{ Right Hemisphere ROI } \\
\hline & \multicolumn{2}{|c|}{ Naming Task } & \multicolumn{2}{|c|}{ Visual-Manual } & \multicolumn{2}{|c|}{ Naming Task } & \multicolumn{2}{|c|}{ Visual-Manual } \\
\hline & Natural & Reversed & Natural & Unnatural & Natural & Reversed & Natural & Unnatural \\
\hline aIPS & -0.03 & $0.10 * * *$ & 0.12 & $0.27 * *$ & -0.03 & $0.15^{* * *}$ & 0.07 & $0.23^{* * *}$ \\
\hline pIPS & -0.22 & $-0.09^{+}$ & 0.08 & $0.26 * *$ & -0.21 & $-0.09 *$ & 0.10 & $0.27^{* * * *}$ \\
\hline FEF & 0.12 & $0.21 * *$ & 0.18 & $0.33 * * *$ & 0.09 & $0.21 * *$ & 0.14 & $0.25^{* * *}$ \\
\hline GFm & -0.09 & $0.02 * *$ & -0.07 & $-0.01^{+}$ & -0.05 & $0.02^{+}$ & -0.09 & $-0.04 n s$ \\
\hline Operculum & 0.07 & $0.16^{*}$ & -0.02 & $0.03 n s$ & 0.21 & $0.38 * * *$ & -0.01 & $0.05 n s$ \\
\hline Precuneus & & & & & -0.35 & $-0.25^{+}$ & 0.03 & $0.26 * *$ \\
\hline GFi & 0.12 & $0.25 * * *$ & 0.07 & $0.18^{*}$ & & & A & \\
\hline Cerebellum & & & & & 0.34 & 0.32 ns & 0.18 & $0.29 * *$ \\
\hline
\end{tabular}

$n s=$ nonsignificant; $\mathrm{N} / \mathrm{A}=$ nonapplicable.

${ }^{+} p<.10$.

$* p<.05$.

$* * p<.01$.

$* * * p<.001$. 
inhibition of prepotent responses or spatial remapping. This subtraction led to highly significant activation in the bilateral IPS and FEFs, and weaker but significant activation in the lateral frontal regions, the same regions activated in the experiments reported here. Thus, we believe that the regions seen here in our study are the ones that play important roles in RS, whether inhibition of prepotent response or spatial remapping is involved or not. An unanswered question is whether the RS regions are also activated by processes related to RS, such as inhibition of prepotent responses and increased working memory load. Further experiments that parametrically manipulate levels of RS difficulty (Schumacher, Elston, \& D'Esposito, 2003) and that isolate each subprocess experimentally are likely to provide more conclusive answers.

Although it is hard to rule out the hypothesis that our activations reflect general difficulty, previous studies suggest that at least some difficult tasks may not always activate the frontal cortex (Schumacher \& D'Esposito, 2002; Owen, 2000; Barch et al., 1997; Demb et al., 1995) and the parietal regions, particularly on the right (Marois, Chun, \& Gore, 2000; Wojciulik \& Kanwisher, 1999). The issue of task difficulty will be directly tested in the companion article (Jiang \& Kanwisher, 2003). There we used two word tasks differing in task difficulty and failed to find increased right parietal activation for the more difficult task. The pattern of activation across the whole brain also differed depending on which type of difficulty was involved. Although task difficulty may be hard to disentangle from the increased demand on specific cognitive processes, it is unlikely that general difficulty can fully account for all common activations seen in our study.

A number of prior imaging studies have tested RS by varying response competition. These include studies using the Stroop color-word naming task (MacLeod, 1991; Stroop, 1935), the Eriksen flanker task (Hazeltine et al., 2000; Botvinick et al., 1999), the anti-saccade and anti-pointing task (Connelly et al., 2000), the go/no-go task (Liddle, Kiehl, \& Smith, 2001; Konishi et al., 1999), and spatial incompatibility tasks (Schumacher \& D'Esposito, 2002; Dassonville et al., 2001; Iacoboni et al., 1996). The areas we saw activated in the current study, namely, the fronto-FEF-parietal network, are also typically seen in many previous studies using other paradigms. For example, in the Stroop task, the brain regions that are more active during incompatible than compatible trials typically include the inferior frontal cortex, the posterior parietal cortex, and the ACC (Zysset, Muller, Lohmann, von Cramon, 2001; Banich et al., 2000; Leung et al., 2000; Bovinick et al., 1999; Carter et al., 1999; Bush et al., 1998; Bench et al., 1993; Pardo et al., 1990). In the flanker task, the lateral prefrontal cortex, the supplementary motor areas, and the parietal cortex show higher BOLD signal on incongruent trials than on congruent trials (Hazeltine et al., 2000; Bunge, Hazeltine,
Scanlon, Rosen, \& Gabrieli, in press). The fronto-FEFparietal network has been implicated in the anti-saccade task (Connolly et al., 2000), the go/no-go task (Liddle et al., 2001; Konishi et al., 1999), and spatial incompatibility tasks (e.g., Schumacher \& D'Esposito, 2002). At the behavioral level, both response competition (with or without inhibition of prepotent responses) and increased mapping complexity are assumed to occupy the central bottleneck (Pashler, 1994). Whether the exact same network is used for different subprocesses of RS remains to be sorted out in future studies that test the same subjects in different paradigms.

\section{Practice Effects}

The persistence of a dual-task interference when two RSs are made is strong evidence that RS is a central cognitive process (Pashler, 1994). Although dual-task interference between two concurrent or adjacent RS tasks is the general rule, this interference can sometimes be reduced or even eliminated after practice (Schumacher et al., 2001; Van Selst, Ruthruff, \& Johnston, 1999). However, even thousands of trials of practice are not always sufficient to eliminate the interference (Ruthruff et al., 2001; Schumacher et al., 2001). Other important conditions for reduced interference are equal emphasis on the two tasks, subject's adoption of a "daring" versus a "conservative" strategy, and highly compatibleS-R mapping in each task (Schumacher et al., 2001). Whether the practice effect is sufficient to reject the existence of a central bottleneck is currently under debate (Levy \& Pashler, 2001; Ruthruff et al., 2001; Van Selst et al., 1999; Meyer \& Kieras, 1997; Pashler, 1994). Future neuroimaging studies may shed light on these questions. For example, one unanswered question concerns the change in neural activation after practice. When dual-task interference from RS is diminished, is the same fronto-FEF-parietal network reported here still recruited, or does extensive training with these tasks lead to the construction of alternate neural pathways dedicated to that task (or combination of tasks)?

\section{Differences among the ROIs}

The co-activation of the frontal, FEF, and parietal ROIs in our tasks, and in many prior studies (Cabeza \& Nyberg, 2000) poses a real challenge for any effort to distinguish between the functions of these regions. However, this co-activation does not mean that these regions carry out identical functions (Hazeltine et al., 2000; Bunge et al., 2002), and may instead reflect the strong anatomical connections between frontal and parietal cortices (Petrides \& Pandya, 1984).

Our data reveal some differences in the pattern of activation across ROIs between the visual-manual task and the verbal naming task. Consider two ROIs, the right 
posterior IPS and the right operculum. In the visualmanual mapping task, the signal increase for incompatible versus compatible RS was high in the right pIPS but low in the right operculum. In contrast, in the verbal naming task, the RS activation was weak in the right pIPS and strong in the right operculum. This three-way interaction between task (visual-manual vs. naming), mapping rule, and region was significant $(p<.03)$. In hindsight, this difference makes sense because the pIPS (extending into the SPL and the precuneus) is typically engaged during spatial processing (Berman et al., 1999; Labar et al., 1999; Culham et al., 1998) while the frontal opercular/insular region falls in the vicinity of Broca's area which has been implicated in linguistic processing (Friederici, Meyer, \& von Cramon, 2000; Price \& Friston, 1997) and tongue movement (Corfield et al., 1999). Thus, RS based on different tasks may not induce identical activation in all ROIs. However, this conclusion is based only on a post hoc analysis that used a lenient statistical criterion $(p<.05$, uncorrected). Converging empirical evidence, especially from techniques with higher temporal resolution, is needed to determine the differences in the contributions made by each of these regions to RS.

To summarize, in three fMRI experiments, we varied the difficulty of mapping a percept onto a response while holding constant the percept and the motor response. Compared with compatible mapping, incompatible $\mathrm{S}-\mathrm{R}$ mapping produced higher BOLD signals in the parietal cortex, the FEF, the cerebellum, and the lateral prefrontal cortex. This activation was seen when the RS was based on visual or auditory inputs, when the mapping was spatial or nonspatial, and when the motor output was manual or verbal. The generality of activation for RS across input and output modalities and across mapping paradigms supports the hypothesis that it constitutes a central cognitive process. However, to qualify as the neural counterpart of the cognitive bottleneck characterized by the behavioral literature (Levy \& Pashler, 1995; Pashler, 1989, 1992), one would demonstrate that these regions are NOT involved in perceptual attention. Thus, it remains to be seen whether any of these regions are selective for RS per se, or whether they have more general functions. This issue will be pursued in the companion article (Jiang \& Kanwisher, 2003).

\section{METHODS}

\section{Subjects}

Twenty-seven subjects between the ages of 18 and 42 participated in these studies (12 women and 15 men). They all had normal or corrected-to-normal vision and normal color vision. Seventeen subjects were tested in Experiment 1, 14 in Experiment 2, and 12 in Experiment 3. Some subjects were scanned in multiple experiments.

\section{Testing Procedure}

Subjects received a 5-min practice in each task on the same day or the day before the scan. They were scanned on a Siemens 3.0 T head-only scanner at the Athinoula A. Martinos Center for Biomedical Imaging in Charlestown, MA. We first acquired 3-D structural images, based on 128 sagittal slices ( $\mathrm{TR}=11 \mathrm{msec}, \mathrm{TE}=4 \mathrm{~mm}$, field of view $[\mathrm{FoV}]=256 \times 256)$, and acquired high resolution T1 images (default TR $=700 \mathrm{msec}, \mathrm{FoV}=200 \times$ $200 \mathrm{~mm}$, resolution $=0.78 \times 0.78 \mathrm{~mm}$ ). Twenty oblique axial slices $6 \mathrm{~mm}$ thick with $0 \mathrm{~mm}$ distance between slices were scanned. The slices extended from the top of the brain to most or all of the cerebellum. We used a T2*-weighted EPI sequence $(\mathrm{TR}=2000 \mathrm{msec}, \mathrm{TE}=$ $30 \mathrm{msec}$, flip angle $=90^{\circ}$, resolution $=3.13 \times 3.13 \times$ $6.00 \mathrm{~mm}$ ) for the functional scans. Each scan lasted $6 \mathrm{~min}$ $4 \mathrm{sec}$; the first $8 \mathrm{sec}$ were discarded. During scanning, the visual display was back projected onto a glass screen, whose image was reflected by a $45^{\circ}$ mirror to the subjects. Auditory stimuli were transmitted by plastic tubes and magnetic-compatible head phones to subjects binaurally. In the visual scans (Experiments 1 and 3), the collection of images was evenly distributed in the 2-sec TR. In the auditory scans (Experiment 2), the collection was bunched to the first $1.312 \mathrm{sec}$ of the 2-sec TR, leaving a 688-msec silent period during which time the auditory stimuli were presented.

\section{Scan Composition}

Each functional scan was a blocked design of three conditions: fixation $(\mathrm{F})$, Task A, and Task B. Each task block lasted $64 \mathrm{sec}$, and each fixation block lasted $20 \mathrm{sec}$. The scan was composed of a series of blocks in which tasks alternated and fixation blocks intervened between each task block. An example of one scan sequence is: "FAFBFAFBF." In all experiments, the two tasks were matched in perceptual input and in motor output. Differences between tasks were introduced by instructions. The first four fixation blocks were each composed of $15 \mathrm{sec}$ fixation followed by $5 \mathrm{sec}$ instruction. The order between Tasks A and B was counterbalanced within subjects as $\mathrm{ABAB}$ or BABA.

\section{Materials and Tasks}

Stimuli were presented using the Psychtoolbox implemented in MATLAB (Brainard, 1997). Presentation of the stimuli was synchronized with scanning using a trigger signal sent by the scanner to the computer.

\section{Experiment 1: Visual-Manual Mapping}

Each trial $(2 \mathrm{sec})$ of the visual-manual task started with a visual display of $100 \mathrm{msec}$, followed by a $100-\mathrm{msec}$ mask, and then a 1800-msec fixation display. Each 
stimulus contained four vertical lines, three of which had identical length whereas one was either shorter or longer than the others. The longer line of each display was either $3.1^{\circ}$ or $2.8^{\circ}$, whereas the shorter line was either $1.0^{\circ}$ or $0.8^{\circ}$. The stimuli were presented against a mid-gray background. The lines were evenly spaced on a $6.25^{\circ}$ by $6.25^{\circ}$ display (Figure 1 ). The mask was made of 18 vertical and 18 horizontal lines (length $=6.25^{\circ}$ ) semiirregularly displaced.

The task on each trial was to identify the line with unique length and report its spatial position among the four lines by pressing one of four keys. Subjects rested their index, middle, ring, and little fingers of the right hand on keys \#1,2,3, and 4. The target position was mapped onto the keys according to a natural mapping rule or an unnatural mapping rule. The instructions preceding the natural mapping blocks schematically illustrated a straightforward mapping (Figure 1, left). The instructions preceding the unnatural mapping informed subjects to press key \#3, 4, 1, and 2 for target at positions 1, 2, 3, and 4, respectively. Each subject completed four scans.

\section{Experiment 2: Auditory-Manual Mapping}

The auditory task was designed to be as similar as possible to the visual task. On each trial, subjects were presented with a sequence of four tones, lasting a total of $480 \mathrm{msec}$. Three of the tones were identical and the other one was unique in duration (or frequency for some subjects). In the frequency task, each tone lasted $45 \mathrm{msec}$, with a blank interval of $100 \mathrm{msec}$ between adjacent tones. The frequency of the higher tone of each trial was either 2000 or $1800 \mathrm{~Hz}$, and the frequency of the lower tone was either 500 or $450 \mathrm{~Hz}$. Each tone was a sine wave with a rise and fall period of $5 \mathrm{msec}$. Subjects were instructed to identify the tone with the unique frequency and report its temporal position within the series by pressing one of four keys. In the duration task, the frequency of all the tones was fixed at $1000 \mathrm{~Hz}$. Three of the tones had short duration (20 $\mathrm{msec})$ while the unique tone was long $(120 \mathrm{msec})$; an interval of 100 msec separated consecutive tones. Subjects were to report the temporal position of the longer tone. Of the 14 subjects, the first 6 were tested in the frequency task and the last 8 were tested in the duration task. The dimension of judgment was changed because the frequency task was reported to be too difficult. Data from the duration and frequency tasks were pooled together because analyses of each task alone showed that the two tasks produced very similar mapwise activation. Furthermore, in the ROI analysis when subject group (frequency vs. duration group) was entered as a between-subject factor, it failed to show any significant main effect or interaction with other factors in any of the ROIs. Only pooled data are reported in the results here.
Subjects were scanned in a blocked design identical to that of Experiment 1, and performed either compatible or incompatible mapping. Each subject received either four or two functional scans (in $\mathrm{ABAB}$ and BABA order). The 480-sec sequence was presented in the middle of the 688-msec silent period in the bunched scanning sequence. Subjects wore earplugs to reduce the loudness of the scanner noise. The loudness of the tones was individually adjusted. The typical loudness was $100 \mathrm{~dB}$ before the dampening (of about $30 \mathrm{~dB}$ ) by the ear plugs and the foam padding.

\section{Experiment 3: Verbal Color-Matching Task}

On each trial two color patches (diameter $=0.93 \mathrm{~cm}$ ), each presented for $100 \mathrm{msec}$ and separated by a 100 -msec interval, were presented at fixation. Subjects were asked to judge whether the colors were identical or different. The colors were chosen from two shades of green (RGB values $\left[\begin{array}{lll}0 & 255 & 0\end{array}\right]$ and $\left.\left[\begin{array}{lll}0 & 255 & 30\end{array}\right]\right)$ and two shades of blue (RGB values [ $\left[\begin{array}{lll}0 & 0 & 255\end{array}\right]$ and $\left[\begin{array}{lll}0 & 30 & 255\end{array}\right]$ ). Half of the trials were match trials, that is, the two colors were identical. The other half were nonmatch trials, one was blue and the other was green.

Subjects were required to make an overt verbal report by saying "same" or "different" while minimizing lip and mouth movement. The instructions were "say SAME when the colors are the same, say DIFFERENT when the colors are different" during the compatible blocks; and "say DIFFERENT when the colors are the same, say SAME when the colors are different" during the incompatible blocks. Verbal responses were not recorded in the scanner. To measure the speed of subjects' response, subjects were instructed to press a key with their right index finger simultaneously with their verbal response, regardless of which verbal response they made. Behavioral studies have shown that when two responses are made to the same feature they do not interfere with each other (Fagot \& Pashler, 1992). Thus, adding the constant task of pressing an index finger should not interfere with verbal responses. In addition, because the same finger responses were made in all conditions, the effect of pushing a key could be subtracted out. Each subject performed two scans in this task; the blocked design and the scanning parameters were identical to those of Experiment 1.

\section{fMRI Data Analysis}

Data were analyzed using SPM99 (http://www.fil.ion. ucl.ac.uk/spm/spm99.html). Each subject's data were motion corrected and then normalized onto a common brain space (the MNI template). Data were then smoothed with a gaussian filter (full width half maximum $=8 \mathrm{~mm}$ ), and high-pass filtered during analysis. We also performed the SPM analysis on unsmoothed data, to make sure that the common activation we found 
across experiments was not due to spatial smoothing. Results based on unsmoothed data were qualitatively similar to those on smoothed data reported here.

We carried out a random effects analysis $(p<.001$, uncorrected for Experiment 1 based on one half of each subject's data, and $p<.005$, uncorrected for Experiments 2 and 3). The threshold was set to be relatively lenient to reduce Type II error. Possible Type I error was further controlled by the ROIs analysis on independent data sets, as follows.

A test of the functional properties of the active regions found in Experiment 1 was performed using an ROI analysis. In Experiment 1, the four scans of each subject were divided into two sets of two scans, each set was counterbalanced in presentation order $(\mathrm{ABAB}$ or $\mathrm{BABA})$. One set of the data were entered in the random effects analysis, and defined group ROIs functionally. Each group ROI was centered on the local maximum of the random effect analysis, with a sphere including active voxels. Regions containing fewer than five significant voxels were not considered. The distance between the center of any two ROIs was at least $12 \mathrm{~mm}$ apart. Each group ROI was within a spherical volume containing the significant voxels; the radius of the ROIs was between 6 and $12 \mathrm{~mm}$, with the constraint that different ROIs did not overlap. Because these group ROIs were generated based on a random effects analysis, they permitted generalization to the population at large. Results focused on the ROIs defined by the visual-manual task because it tested a large number of subjects on multiple scans. We also conducted ROI analysis on ROIs defined by the auditory-manual or the color-verbal task and found similar activation across experiments.

PSC relative to fixation baseline $[$ PSC $=100 *$ raw BOLD magnitude for (the task - fixation)/raw BOLD magnitude for fixation] was calculated for both each mapping condition within each ROI for each subject. These values were then entered in an ANOVA; the significance of the ANOVA was reported in each table. Because the data defining the ROIs were independent from data used to calculate PSCs, Type I errors were drastically reduced.

\section{Acknowledgments}

This work was supported by a Human Frontiers' grant to N.K. Y.J. was supported by a research fellowship from the Helen Hay Whitney Foundation. We thank Miles Shuman for technical assistance and Kyungmouk Lee for data analysis, and Silvia Bunge, Mark D'Esposito, John Duncan, Molly Potter, Rebecca Saxe, and Eric Schumacher for helpful comments on the manuscript.

Reprint requests should be sent to Yuhong Jiang, currently at the Department of Psychology, Harvard University, 33 Kirkland St., Room 820, Cambridge, MA 02138, USA, or via e-mail: yuhong@wjh.harvard.edu.
The data reported in this experiment have been deposited in the fMRI Data Center (http://www.fmridc.org). The accession number is 2-2003-113T5.

\section{REFERENCES}

Banich, M. T., Milham, M. P., Atchley, R. A., Cohen, N. J., Webb, A., Wszalek, T., Kramer, A. F., Liang, Z.-P., Wright, A., Shenker, J., Magin, R., Barad, V., Gullett, D., Shah, C., \& Brown, C. (2000). fMRI studies of Stroop tasks reveal unique roles of anterior and posterior brain systems in attentional selection. Journal of Cognitive Neuroscience, 12, 988-1000.

Barch, D. M., Braver, T. S., Akbudak, E., Conturo, T., Ollinger, J., \& Snyder, A. (2001). Anterior cingulate cortex and response conflict: Effects of response modality and processing domain. Cerebral Cortex, 11, 837-848.

Barch, D. M., Braver, T. S., Nystrom, L. E., Forman, S. D., Noll, D. C., \& Cohen, J. D. (1997). Dissociating working memory from task difficulty in human prefrontal cortex.

Neuropsychologia, 35, 1373-1380.

Barch, D. M., Braver, T. S., Sabb, F. W., \& Noll, D. C. (2000). Anterior cingulate and the monitoring of response conflict: Evidence from an fMRI study of overt verb generation. Journal of Cognitive Neuroscience, 12, 298-309.

Barch, D. M., Sabb, F. W., Carter, C. S., Braver, T. S., Noll, D. C., \& Cohen, J. D. (1999). Overt verbal responding during fMRI scanning: Empirical investigations of problems and potential solutions. Neuroimage, 10, 642-657.

Beauchamp, M. S., Haxby, J. V., Jennings, J. E., \& DeYoe, E. A. (1999). An fMRI version of the Farnsworth-Munsell 100-Hue test reveals multiple color-selective areas in human ventral occipitotemporal cortex. Cerebral Cortex, 9, 257-263.

Bench, C. J., Frith, C. D., Grasby, P. M., Friston, K. J., Paulesu, E., Frackowiak, R. S., \& Dolan, R. J. (1993). Investigations of the functional anatomy of attention using the Stroop test. Neuropsychologia, 31, 907-922.

Berman, R. A., Colby, C. L., Genovese, C. R., Voyvodic, J. T., Luna, B., Thulborn, K. R., \& Sweeney, J. A. (1999). Cortical networks subserving pursuit and saccadic eye movements in humans: An fMRI study. Human Brain Mapping, 8, $209-225$.

Borger, R. (1963). The refractory period and serial choice reactions. Quarterly Journal of Experimental Psychology, $15,1-12$.

Botvinick, M., Nystrom, L. E., Fissell, K., Carter, C. S., \& Cohen, J. D. (1999). Conflict monitoring versus selection-for-action in anterior cingulate cortex. Nature, 402, 179-181.

Brainard, D. H. (1997). The psychophysics toolbox. Spatial Vision, 10, 433-436.

Bunge, S. A., Hazeltine, E., Scanlon, M. D., Rosen, A. C., \& Gabrieli, J. D. E. (2002). Dissociable contributions of prefrontal and parietal cortices to response selection. Neuroimage, 17, 1562-1571.

Bush, G., Whalen, P. J., Rosen, B. R., Jenike, M. A., McInerney, S. C., \& Rauch, S. L. (1998). The counting Stroop: An interference task specialized for functional neuroimaging - validation study with functional MRI. Human Brain Mapping, 6, 270-282.

Cabeza, R., \& Nyberg, L. (2000). Imaging cognition: II. An empirical review of 275 PET and fMRI studies. Journal of Cognitive Neuroscience, 12, 1- 47.

Carter, C. S., Botvinick, M. M., \& Cohen, J. D. (1999). The contribution of the anterior cingulate cortex to executive processes in cognition. Reviews in the Neurosciences, 10, 49-57.

Carter, C. S., Braver, T. S., Barch, D. M., Botvinick, M. M., Noll, 
D., \& Cohen, J. D. (1998). Anterior cingulate cortex, error detection, and the online monitoring of performance. Science, 280, 747-749.

Connolly, J. D., Goodale, M. A., Desouza, J. F., Menon, R. S., \& Vilis, T. (2000). A comparison of frontoparietal fMRI activation during anti-saccades and anti-pointing. Journal of Neurophysiology, 84, 1645-1655.

Corfield, D. R., Murphy, K., et al. (1999). Cortical and subcortical control of tongue movement in humans: A functional neuroimaging study using fMRI. Journal of Applied Physiology, 86, 1468-1477.

Culham, J. C., Brandt, S. A., Cavanagh, P., Kanwisher, N. G., Dale, A. M., \& Tootell, R. B. (1998). Cortical fMRI activation produced by attentive tracking of moving targets. Journal of Neurophysiology, 80, 2657-2670.

Dassonville, P., Lewis, S. M., Zhu, X. H., Ugurbil, K., Kim, S. G., Ashe, J. (2001). The effect of stimulus-response compatibility on cortical motor activation. Neuroimage, 13, $1-14$

Demb, J. B., Desmond, J. E., Wagner, A. D., Vaidya, C. J., Glover, G. H., \& Gabrieli, J. D. (1995). Semantic encoding and retrieval in the left inferior prefrontal cortex: A functional MRI study of task difficulty and process specificity. Journal of Neuroscience, 15, 5870-5878.

Duncan, J., Martens, S., \& Ward, R. (1996). Restricted attentional capacity within but not between sensory modalities. Nature, 379, 808-810.

Fagot, C., \& Pashler, H. (1992). Making two responses to a single object: Implications for the central attentional bottleneck. Journal of Experimental Psychology: Human Perception and Performance, 18, 1058-1079.

Friederici, A. D., Meyer, M., \& von Cramon, D. Y. (2000). Auditory language comprehension: An event-related fMRI study on the processing of syntactic and lexical information. Brain and Language, 75, 289-300.

Gusnard, D. A., \& Raichle, M. E. (2001). Searching for a baseline: Functional imaging and the resting human brain. Nature Reviews Neuroscience, 2, 685-694.

Hazeltine, E., Poldrack, R., \& Gabrieli, J. D. (2000). Neural activation during response competition. Journal of Cognitive Neuroscience, 12, 118-129.

Huang, J., Carr, T. H., \& Cao, Y. (2002). Comparing cortical activations for silent and overt speech using event-related fMRI. Human Brain Mapping, 15, 39-53.

Iacoboni, M., Woods, R.P., \& Mazziotta, J.C. (1996). Brainbehavior relationships: Evidence from practice effects in spatial stimulus-response compatibility. Journal of Neurophysiology, 76, 321-331.

Jiang, Y., \& Kanwisher, N. (2003). Common neural mechanism for response selection and perceptual processing. Journal of Cognitive Neuroscience, 15, 1095-1110.

Jiang, Y., \& Kanwisher, N. (in preparation). Involvement of the parietal-frontal regions in nonspatial response selection.

Konishi, S., Nakajima, K., Uchida, I., Kikyo, H., Kameyama, M., \& Miyashita, Y. (1999). Common inhibitory mechanism in human inferior prefrontal cortex revealed by event-related functional MRI. Brain, 122, 981-991.

LaBar, K. S., Gitelman, D. R., Parrish, T. B., Mesulam, M. (1999). Neuroanatomic overlap of working memory and spatial attention networks: A functional MRI comparison within subjects. Neuroimage, 10, 695-704.

Leung, H. C., Skudlarski, P., Gatenby, J. C., Peterson, B. S., \& Gore, J. C. (2000). An event-related functional MRI study of the Stroop color word interference task. Cerebral Cortex, $10,552-560$.

Levy, J., \& Pashler, H. (1995). Does perceptual analysis continue during selection and production of a speeded response? Acta Psychologica, 90, 245-260.
Levy, J., \& Pashler, H. (2001). Is dual-task slowing instruction dependent. Journal of Experimental Psychology: Human Perception and Performance, 27, 862-869.

Liddle, P. F., Kiehl, K. A., \& Smith, A. M. (2001). Event-related fMRI study of response inhibition. Human Brain Mapping, $12,100-109$

MacLeod, C. M. (1991). Half a century of research on the Stroop effect: An integrative review. Psychological Bulletin, 109, 163-203.

Marois, R., Chun, M. M., \& Gore, J. C. (2000). Neural correlates of the attentional blink. Neuron, 28, 299-308.

McCann, R. S., \& Johnston, J. C. (1992). Locus of the single-channel bottleneck in dual-task interference. Journal of Experimental Psychology: Human Perception and Performance, 18, 471-484.

Meyer, D. E., \& Kieras, D. E. (1997). A computational theory of executive cognitive processes and multiple-task performance: Part 2. Accounts of psychological refractory-period phenomena. Psychological Review, 104, $749-791$.

Meyer, D. E., Kieras, D. E., Lauber, E., Schumacher, E. H., Glass, J., Zurbriggen, E., Gmeindl, L., \& Apfelblat, D. (1995). Adaptive executive control: Flexible multiple-task performance without pervasive immutable response-selection bottlenecks. Acta Psychologica, 90, $163-190$.

Owen, A. M. (2000). The role of the lateral prefrontal cortex in mnemonic processing: The contribution of functional neuroimaging. Experimental Brain Research, 133, 33-43.

Palmer, E. D., Rosen, H. J., Ojemann, J. G., Buckner, R. L., Kelley, W. M., Petersen, S. E. (2001). An event-related fMRI study of overt and covert word stem completion. Neuroimage, 14, 182-193.

Pardo, J. V., Pardo, P. J., Janer, K. W., \& Raichle, M. E. (1990). The anterior cingulate cortex mediates processing selection in the Stroop attentional conflict paradigm. Proceedings of the National Academy of Sciences, U.S.A., 87, 256-259.

Pashler, H. (1984). Processing stages in overlapping tasks: Evidence for a central bottleneck. Journal of Experimental Psychology: Human Perception and Performance, 10, 358-377.

Pashler, H. (1989). Dissociations and dependencies between speed and accuracy: Evidence for a two-component theory of divided attention in simple tasks. Cognitive Psychology, 21, 469-514.

Pashler, H. (1990). Do response modality effects support multiprocessor models of divided attention? Journal of Experimental Psychology: Human Perception and Performance, 16, 826-840.

Pashler, H. (1992). Shifting visual attention and selecting motor responses: Distinct attentional mechanisms. Journal of Experimental Psychology: Human Perception and Performance, 17, 1023-1040.

Pashler, H. (1994). Dual-task interference in simple tasks: Data and theory. Psychological Bulletin, 116, 220-244.

Pashler, H. (1998). The Psychology of Attention. Cambridge: MIT Press.

Pashler, H., Carrier, M., \& Hoffman, J. (1993). Saccadic eye movements and dual-task interference. Quarterly Journal of Experimental Psychology, 46A, 51-82.

Petrides, M., \& Pandya, D. N. (1984). Projections to the frontal cortex from the posterior parietal region in the rhesus monkey. Journal of Comparative Neurology, 228, 105-116.

Price, C. J., \& Friston, K. J. (1997). The temporal dynamics of reading: A PET study. Proceedings of the Royal Society of London, B: Biological Sciences, 264, 1785-1791.

Raichle, M. E., MacLeod, A. M., Snyder, A. Z., Powers, W. J., 
Gusnard, D. A., \& Shulman, G. L. (2001). A default mode of brain function. Proceedings of the National Academy of Science, U.S.A., 98, 676-682.

Rosen, H. J., Ojemann, J. G., Ollinger, J. M., \& Petersen, S. E. (2000). Comparison of brain activation during word retrieval done silently and aloud using fMRI. Brain and Cognition, 42, 201-217.

Rushworth, M. F. S., Hadland, K. A., Paus, T., \& Sipila, P. K. (2001). Role of the human medial frontal cortex in task switching: A combined fMRI and TMS study. Journal of Neurophysiology, 87, 2577-2592.

Ruthruff, E., Pashler, H., \& Klaassen, A. (2001). Processing bottlenecks in dual-task performance: Structural limitation or strategic postponement? Psychonomic Bulletin and Review, 8, 73-80.

Schumacher, E. H., \& D'Esposito, M. (2002). Neural implementation of response selection in humans as revealed by localized effects of stimulus-response compatibility on brain activation. Human Brain Mapping, 17, 193-201.

Schumacher, E. H., Elston, P. H., \& D'Esposito, M. (2003). Neural evidence for representation-specific response selection. Journal of Cognitive Neuroscience, 15, 1111-1121.

Schumacher, E. H., Lauber, E. J., Glass, J. M., Zurbriggen, E. L., Gmeindl, L., Kieras, D. E., \& Meyer, D. E. (1999). Concurrent response-selection processes in dual-task performance: Evidence for adaptive executive control of task scheduling. Journal of Experimental Psychology: Human Perception and Performance, 25, 791-814.

Schumacher, E. H., Seymour, T. L., Glass, J. M., Kieras, D. E., \& Meyer, D. E. (2001). Virtually perfect time sharing in dual-task performance: Uncorking the central attentional bottleneck. Psychological Science, 12, 101-108.

Shulman, G. L., Fiez, J. A., Corbetta, M., Buckner, R. L., Miezin, F. M., Raichle, M. E., \& Petersen, S. E. (1997). Common blood flow changes across visual tasks: II. Decreases in cerebral cortex. Journal of Cognitive Neuroscience, 9, 648-663.

Stroop, J. R. (1935). Studies of interference in serial verbal reactions. Journal of Experimental Psychology, 18, 643-662.

Tootell, R. B., \& Hadjikhani, N. (2001). Where is "dorsal V4" in human visual cortex? Retinotopic, topographic and functional evidence. Cerebral Cortex, 11, 298-311.

Treisman, A., \& Davies, A. (1973). Dividing attention to ear and eye. In S. Kornblum (Ed.), Attention and performance IV (pp. 101-107). New York: Academic Press.

Van Selst, M., Ruthruff, E., \& Johnston, J. C. (1999). Can practice eliminate the psychological refractory period effect? Journal of Experimental Psychology: Human Perception and Performance, 25, 1268-1283.

Van Veen, V., Cohen, J. D., Botvinick, M. M., Stenger, V. A., \& Carter, C. S. (2001). Anterior cingulate cortex, conflict monitoring, and levels of processing. Neuroimage, 14, $1302-1308$.

Wojciulik, E., \& Kanwisher, N. (1999). The generality of parietal involvement in visual attention. Neuron, 23, $747-764$.

Zysset, S., Muller, K., Lohmann, G., \& von Cramon, D. Y. (2001). Color-word matching stroop task: Separating interference and response conflict. Neuroimage, 13, 29-36. 